\title{
Quantitative Relationships between Huntingtin Levels, Polyglutamine Length, Inclusion Body Formation, and Neuronal Death Provide Novel Insight into Huntington's Disease Molecular Pathogenesis
}

\author{
Jason Miller, ${ }^{1,2,3,4}$ Montserrat Arrasate, ${ }^{1,2}$ Benjamin A. Shaby, ${ }^{9}$ Siddhartha Mitra, ${ }^{1,4,6}$ Eliezer Masliah, ${ }^{10,11}$ \\ and Steven Finkbeiner ${ }^{1,2,5,6,7,8}$ \\ ${ }^{1}$ Gladstone Institute of Neurological Disease, ${ }^{2}$ Taube-Koret Center for Huntington's Disease Research, ${ }^{3}$ Chemistry and Chemical Biology Program, \\ ${ }^{4}$ Medical Scientist Training Program, and ${ }^{5}$ Neuroscience Program, University of California, San Francisco, California 94158, ${ }^{6}$ Biomedical Sciences \\ Program, and Departments of ${ }^{7}$ Neurology and ${ }^{8}$ Physiology, University of California, San Francisco, California 94143, ${ }^{9}$ Department of Statistical Science, \\ Duke University, Durham, North Carolina 27708, and Departments of ${ }^{10}$ Neurosciences and ${ }^{11}$ Pathology, University of California, San Diego, La Jolla, \\ California 92093
}

An expanded polyglutamine (polyQ) stretch in the protein huntingtin (htt) induces self-aggregation into inclusion bodies (IBs) and causes Huntington's disease (HD). Defining precise relationships between early observable variables and neuronal death at the molecular and cellular levels should improve our understanding of HD pathogenesis. Here, we used an automated microscope that tracks thousands of neurons individually over their entire lifetime to quantify interconnected relationships between early variables, such as htt levels, polyQ length, and IB formation, and neuronal death in a primary striatal model of HD. The resulting model revealed that mutant htt increases the risk of death by tonically interfering with homeostatic coping mechanisms rather than producing accumulated damage to the neuron, htt toxicity is saturable, the rate-limiting steps for inclusion body formation and death can be traced to different conformational changes in monomeric htt, and IB formation reduces the impact of the starting levels of htt of a neuron on its risk of death. Finally, the model that emerges from our quantitative measurements places critical limits on the potential mechanisms by which mutant htt might induce neurodegeneration, which should help direct future research.

\section{Introduction}

Huntington's disease (HD) is a progressive, uniformly fatal neurodegenerative condition caused by a polyglutamine (polyQ) expansion in the protein huntingtin (htt). Expansions of $>36-39$ trigger disease, and longer polyQ stretches above this threshold cause earlier disease onset. The polyQ expansion causes marked striatal atrophy and induces htt to aggregate into protein deposits termed inclusion bodies (IBs) (Orr and Zoghbi, 2007).

Received Jan. 9, 2010; revised May 21, 2010; accepted June 11, 2010.

This work was supported by the National Institutes of Health (NIH)-National Institute of General Medical Sciences University of California, San Francisco (UCSF), Medical Scientist Training Program and a fellowship from the UCSF Hilblom Center for the Biology of Aging (J.M.). E.M. was supported, in part, by NIH Grants AG18440, AG11385, and AG022074. S.F. was supported by the Taube-Koret Center for Huntington's Disease Research, by NIH-National Institute of Neurological Disorders and Stroke Grants 2R01 NS039074 and 2R01 NS045091 and NIH-National Institute on Aging Grant 2P01 AG022074, and by The J. David Gladstone Institutes. The animal care facility was partly supported by an NIH Extramural Research Facilities Improvement Program Project (Grant C06 RR018928). We thank A. Kazantzev and D. Housman for the htt CDNAs and R. Tsien for the mRFP cDNA that we used to construct our mammalian expression plasmids. We thank members of the Finkbeiner Laboratory for useful discussions, G. Howard and S. Ordway for editorial assistance, and K. Nelson for administrative assistance.

Correspondence should be addressed to Steven Finkbeiner, Gladstone Institute of Neurological Disease, 1650 Owens Street, San Francisco, California 94158. E-mail: sfinkbeiner@gladstone.ucsf.edu.

M. Arrasate's present address: Division of Neuroscience, Centro de Investigación Médica Aplicada, University of Navarra, Avenida Pío XIl, 55, E-31008 Pamplona, Spain.

DOI:10.1523/JNEUROSCI.0146-10.2010

Copyright $\odot 2010$ the authors $\quad$ 0270-6474/10/3010541-10\$15.00/0
A number of controversies about HD pathogenesis remain difficult to resolve. For example, is the rate-limiting step in IB formation a monomeric change in mutant htt conformation (Chen et al., 2002) or the collision of two htt molecules (Colby et al., 2006)? Does mutant htt obstruct the protein quality control systems of a neuron (Bennett et al., 2005), or is it efficiently degraded by these systems (Michalik and Van Broeckhoven, 2004)? To resolve these questions, hypotheses must be tested against a dataset in which basic variables (e.g., htt levels, polyQ length, IB formation, and neuronal death) are measured in living neurons and quantitatively related to each other. For example, if IB formation requires the collision of two htt molecules, then IB formation should display a second-order dependence on the htt concentration of a neuron. If htt tonically obstructs the protein quality control systems of the neuron in a polyQ-dependent fashion, steady-state levels of htt may increase with longer polyQ lengths.

Unfortunately, determining cause-effect relationships between cellular variables in HD is fraught with difficulty. Disease states have generally not been honed by evolutionary pressure, so they often defy the mechanistic logic of physiological processes. Although an observed process in a physiologic system can often be presumed to be important in that system, an observed abnormality in a disease state may be a cause, cellular response, or 
incidental to the disease. Time-course studies can suggest causeeffect relationships between abnormalities and disease phenotypes in cell-based experimental systems. However, such studies usually measure an average value from a population of cells, masking complicated relationships that would emerge if a large number of single-cell, time-course observations were made (Finkbeiner et al., 2006). In addition, the qualitative nature of most cellular disease-related research makes it difficult to determine whether the degree of a particular abnormality is enough to account for a disease phenotype. For example, an abnormality may precede and positively correlate with an outcome, but the kinetics of the abnormality and the outcome may not match.

In this study, we applied an automated microscopy system to a primary striatal neuron model of HD to establish quantitative, temporal relationships among htt levels, polyQ length, IB formation, and neuronal death. Our approach overcomes the limitations of conventional cellular disease-oriented methods for determining cause-effect relationships. The relationships we quantify establish a basic cellular "systems" model of HD.

\section{Materials and Methods}

Plasmids. Expression plasmids encoding an N-terminal fragment of htt fused to enhanced green fluorescent protein (eGFP) [pGW1-htt ${ }^{\mathrm{ex} 1}-\left(\mathrm{Q}_{46}\right.$ or $\mathrm{Q}_{97}$ )-eGFP] were derived from pcDNA3.1-based plasmids by subcloning into pGW1-CMV (British Biotechnology). Plasmid constructs were confirmed by sequencing. $\mathrm{Htt}^{\mathrm{exl}}-\left(\mathrm{Q}_{17}, \mathrm{Q}_{72}\right)$-eGFP, pGW1-eGFP, and pGW1-mRFP have been described (Arrasate et al., 2004). The polyQ stretch for all htt constructs was encoded by irregularly alternating CAG and CAA codons.

Primary striatal culture. Primary cultures of rat striatal neurons were prepared from embryos [embryonic day 18 (E18) to E20] and transfected with plasmids (4-6 d in vitro) as described (http://www. gladstone.ucsf.edu/gladstone/site/finkbeiner/section/1193). Typically, neurons were cotransfected with pGW1-mRFP and a version of pGW1-htt ${ }^{\text {ex }}$ $\left(\mathrm{Q}_{17}, \mathrm{Q}_{47}, \mathrm{Q}_{72}\right.$, or $\left.\mathrm{Q}_{97}\right)$-eGFP in a $\sim 1: 1$ molar ratio, with a total of 1-4 $\mu \mathrm{g}$ of DNA in each well of a 24-well plate. After transfection, neurons were maintained in serum-free medium.

Immunocytochemistry, confocal microscopy, and electron microscopy. Immunocytochemistry was performed as described (Brooks et al., 2004) using a $12 \mathrm{~min} 4 \%$ paraformaldehyde/ $4 \%$ sucrose fixation. Images were collected on a Zeiss LSM 510 confocal microscope with Zeiss software (Zeiss). For electron microscopy, primary striatal cell cultures were plated in glass-bottom dishes (MatTek). Plates were fixed in 4\% paraformaldehyde for $15 \mathrm{~min}$. Cells were then further fixed in $2 \%$ paraformaldehyde and $1 \%$ glutaraldehyde, and then fixed in osmium tetraoxide and embedded in Epon Araldite. Once the resin hardened, blocks with the cells were detached from the coverslips and mounted into a resin block for sectioning with an ultramicrotome (Leica) at $90 \mathrm{~nm}$ thickness. Grids containing the attached ultrathin sections were analyzed with a Zeiss OM 10 electron microscope (Zeiss). Cells were randomly acquired from three grids.

Robotic microscope imaging system. All longitudinal microscopy experiments were performed on an automated microscopy system as described previously (Arrasate et al., 2004; Arrasate and Finkbeiner, 2005; Mitra et al., 2009).

Image analysis and statistics. Measurements of htt expression, IB formation, and survival were extracted from files generated with automated imaging by automated analysis programs or by visual inspection as described (Arrasate et al., 2004; Mitra et al., 2009). Levels of diffuse htt ${ }^{\text {exl }}$ $\mathrm{Q}_{n}$-eGFP (i.e., fluorescence outside of IBs) were made using measurements of fluorescence of the eGFP tag from images of live cells, which is an accurate marker for diffuse htt levels in a given neuron (Arrasate et al., 2004). Methods for detecting IBs in live neurons have also been described and validated (Arrasate et al., 2004). The abrupt loss of a cotransfected fluorescent protein [e.g., monomeric red fluorescent protein (mRFP)], a validated marker of cell death (Arrasate et al., 2004), was used to estimate the survival time of each neuron. For actuarial (see Fig. 2a) and Cox analysis (see Fig. 3), time to death was defined as the imaging time point after transfection that the neuron was last seen alive. For Weibull analysis (see Fig. 4), time to death was defined as an unknown time point within the interval range bounded by the last imaging time point after transfection that the neuron was seen alive and the first imaging time point the neuron was no longer seen. We define neurodegeneration in our model system to be synonymous with the death of transfected neurons, as measured by loss of the cotransfection marker fluorescence.

$\mathrm{Htt}$ expression was measured $24 \mathrm{~h}$ after transfection. Neurons that had already formed IBs at $24 \mathrm{~h}$ were excluded from all analysis that required the measurement of diffuse htt levels at $24 \mathrm{~h}$. We confirmed that htt levels in neurons that have not formed an IB $24 \mathrm{~h}$ after transfection are directly proportional to average htt levels in those same neurons up until the neuron forms an IB or dies (J. Miller and S. Finkbeiner, unpublished observations). Actuarial analysis (see Fig. $2 a$ ) was calculated using StatView software (SAS Institute). The 95\% confidence intervals for the rest of Figure 2 were calculated in Prism (GraphPad).

Cox regression analysis in Figure 3 was performed using the survival package in R. Hazards were regressed on htt or eGFP levels using penalized splines (pspline in R). The Cox model included htt or eGFP levels as the continuous covariate, whereas stratification variables included experiment date and whether the neuron formed an inclusion body at any point during the experiment (full model). All Cox analyses were repeated treating experiment date as a nominal covariate instead, with results comparable with the full model. Additionally, all Cox analyses were repeated on just the cohort of neurons that never formed an IB during the course of the experiment, again with results comparable with the full model.

Each Cox model was analyzed for violations of proportional hazards and for influential datapoints with the cox.zph and dfbeta functions in R, respectfully. Only the eGFP-only data violated proportional hazards in a traditional Cox model, but the penalized spline version of the model, as it appears in Figure 3, no longer locally or globally violates proportional hazards. Two of 524 neurons were deemed to be overly influential for the $\mathrm{Q}_{17}$ dataset [defined, using the dfbeta function in $\mathrm{R}$, as a single neuron changing the absolute value of the Cox coefficient by at least $25 \%$; this definition is conservative compared with other published studies (for example, see Dominici et al., 2007)]. Two of 558 neurons were deemed, by the same criteria, to be overly influential for the $\mathrm{Q}_{72}$ dataset. One of 439 neurons was deemed to be overly influential for the $Q_{97}$ dataset. Overly influential neurons were eliminated from the datasets presented in final form in Figure 3. Comparing the Cox analysis with and without the overly influential neurons, the trends (and overall conclusions from Fig. 3) are the same but are more significant (higher Cox coefficient value, lower $p$ value) when the influential neurons are removed. Thus, the $\mathrm{Q}_{17}, \mathrm{Q}_{72}$, and $\mathrm{Q}_{97}$ models presented in Figure 3 would have slightly lower slopes and slightly higher $p$ values if the influential neurons were included in the datasets.

For the regression analysis in Figure 4, we used a Weibull accelerated failure time model that accommodates interval censored data (available in the survreg function in R). Intervals were defined as the periods between each imaging time (typically $24 \mathrm{~h}$ ). Analysis in Figure 4 was performed with a bivariate Weibull model, including the continuous covariate of htt levels and the nominal covariate of experiment date. A penalized spline was used to model the "htt levels" covariate. The assumptions implicit in the Weibull model (namely, log-Weibull distributed failure times and a proportional acceleration of failure time by htt levels) were tested graphically. Influential datapoints were tested for using dfbeta in R, but no datapoints were deemed overly influential. The analysis of IB formation rate included all neurons; those that did not form an IB during the experiment (either because death intervened or the neurons survived without an IB to the end of the experiment) were right censored. The analysis of "death rate without IB formation" included only those neurons that did not form an IB during the experiment. The analysis of "death rate after IB formation" included only those neurons that did form an IB during the experiment. The $y$-axis for the Figure 4 plots is the negative partial effect of htt from the accelerated failure time model; this value is linearly proportional to rate $[-\ln$ (time to failure) $\sim$ rate of failure time]. 
All figures and tables except Figure $2 c$ represent results from the combination of at least three (and up to eight) different experiments covering hundreds to thousands of neurons.

Quantification of immunofluorescence. Quantitation of immunofluorescence was performed on a Zeiss LSM 510 microscope. Lasers were given $2 \mathrm{~h}$ to warm up and stabilize before any measurements were made. For each fluorescent color to be imaged, a photomultiplier tube (PMT) detector was set to a single gain and offset setting appropriate for imaging the average intensity of that color. To avoid effects of photobleaching, each neuron selected for imaging was illuminated with light for $<5 \mathrm{~s}$ before a single image was acquired. Images were acquired using a $63 \times$, 1.4 numerical aperture Zeiss oil-immersion lens. Levels of fluorescence from the eGFP tag fused to htt, mRFP, and/or a fluorophore-conjugated secondary antibody bound to a primary anti-htt antibody were then extracted from images by drawing a region of interest across the neuronal cell body and recording average pixel intensity. Background subtraction was unnecessary as PMT offset was set to virtually eliminate background signals. Neurons with IBs were excluded from quantification. When combining multiple quantitative immunofluorescence datasets, differences in fluorescence values caused by different detector range settings were normalized.

In some experiments (in Fig. 2c), htt constructs were cotransfected with $\mathrm{mRFP}$ in a DNA ratio of 1:1 into primary striatal neurons. Neurons were fixed at $24 \mathrm{~h}$ after transfection with a $4 \%$ paraformaldehyde $/ 4 \%$ sucrose solution for $12 \mathrm{~min}$. The htt signal in each neuron (measured by eGFP fluorescence) was then normalized to the mRFP signal of that neuron, a cotransfection marker. The expression of htt and the cotransfection marker are driven from identical GW1 plasmids and promoters.

\section{Results}

\section{A validated primary striatal model of HD}

We developed a primary striatal neuron model of HD, described in Figure $1 a$, which recapitulates salient features of HD. These features include the formation of IBs in neurites (Fig. 1b), mostly granular, amorphous IBs (Fig. 1c), and various other IB attributes elaborated in Figure 1d. Like HD, our model also exhibits polyQdependent nuclear fragmentation, neurite dystrophy, and death as well as differential susceptibility of neuronal subtypes to mutant htt (Fig. 1d). Furthermore, the model predicted key findings observed in animal models of polyQ disorders or in HD, including the mitigating effect of IB formation (Fig. 1e). Given the extent to which this model of HD has been validated, we reasoned that a more in-depth quantitative analysis of data from the model could yield additional insights into HD molecular pathogenesis.

\section{Mutant htt imparts an initially constant, then decreasing, risk of death on striatal neurons}

To begin, we examined the rate of neuronal death in our system. This examination was facilitated by our ability to track thousands of individual neurons over their entire lifetimes. If mutant htt causes cumulative damage to neurons, then the rate of neuronal death should increase over time (Clarke et al., 2000). Alternatively, mutant htt may tonically constrain the homeostatic capacity of neurons, making them more susceptible to random "one-hit" events that would not normally overwhelm wild-type neurons (Clarke et al., 2000). In a one-hit model, the rate of neuronal death should be constant over time.

We found that, for the first $5 \mathrm{~d}$ after transfection of mutant htt into striatal neurons, the risk of death in our model system was constant, supporting the one-hit hypothesis (Fig. 2a). Furthermore, the risk of death was tonically higher for neurons with $\mathrm{htt}^{\mathrm{ex} 1}-\mathrm{Q}_{97}$-eGFP than those with $\mathrm{htt}^{\mathrm{ex} 1}-\mathrm{Q}_{46}$-eGFP. This finding is consistent with the idea that increasing polyQ length decreases the homeostatic capacity of a neuron throughout its lifetime. Interestingly, beginning around $6 \mathrm{~d}$ after transfection, the risk of death decreases for both $h t^{\text {exl }}-\mathrm{Q}_{46}-\mathrm{eGFP}$ and $h \mathrm{tt}^{\mathrm{exl}}-\mathrm{Q}_{97}-\mathrm{eGFP}$ neurons, which is inconsistent with the competing cumulative damage hypothesis (Fig. 2a).

\section{Increasing polyQ length results in decreased htt expression but leads to increased abundance of a specific conformation of htt}

We next sought to use our model to shed light on the mechanism of expanded polyQ toxicity. One possibility is that the polyQ stretch stabilizes htt without changing its function. The resulting increase in steady-state htt levels might be inherently toxic to neurons. For example, a polyQ expansion in another protein, ataxin 7 , induces accumulation of the protein, and the resulting increase in normal ataxin 7 function has been proposed to contribute to a neurodegenerative polyQ disorder called SCA7 (spinocerebellar ataxia 7) (Yoo et al., 2003). Alternatively, longer polyQ stretches might change htt function by preferentially stabilizing a toxic isoform of the protein over less toxic isoforms (Nagai et al., 2007).

To distinguish these possibilities, we quantified the relationship between polyQ length and steady-state htt levels. We measured the fluorescence signal from each of hundreds of htt ${ }^{\text {exl }}-\mathrm{Q}_{n}$-eGFPtransfected neurons without IBs at $24 \mathrm{~h}$ after transfection, a time when IB formation is minimal even for the htt ${ }^{\text {ex } 1}-Q_{97}-e G F P$ cohort. In contrast to ataxin 7 , we found an inverse relationship between htt ${ }^{\text {exl }}$ levels and polyQ length (Fig. 2b).

Although the number of neurons excluded from our analysis of polyQ length versus steady-state htt levels was small (i.e., those few neurons in which an IB had already formed by $24 \mathrm{~h}$ after transfection), we wondered whether excluding these neurons might affect our analysis. For example, neurons with higher htt levels and long polyQ stretches form IBs earlier (Arrasate et al., 2004) (J. Miller and S. Finkbeiner, unpublished observations), so we may have preferentially excluded high-expressing neurons in the long polyQ cohort more than the shorter polyQ cohort. To rule out this possibility, we cotransfected neurons with htt ${ }^{\text {exl }}-Q_{17}$ or $97-e G F P$ and mRFP plasmid DNA in a 1:1 ratio. Since all constructs were in the same plasmid backbone with the same promoter, we used the fluorescence of $\mathrm{mRFP}$ as a surrogate marker for the transcriptional activation of all the cotransfected plasmids in each neuron. This allowed us to select neurons transfected with htt ${ }^{\mathrm{ex1}}-\mathrm{Q}_{17^{-}}$ eGFP or htt ${ }^{\text {exl }}-Q_{97}-e G F P$ and without IBs but with comparable gene dosage. In this analysis, diffuse htt levels were still significantly lower in the $\mathrm{htt}^{\mathrm{ex}}{ }_{-}-\mathrm{Q}_{97}$-eGFP-transfected neurons (Fig. $2 c$ ). Therefore, the inverse relationship between diffuse htt levels and polyQ length is not simply a by-product of faster IB formation in longer polyQ-htt-transfected neurons.

Our results demonstrate that the polyQ stretch of htt does not lead to increased steady-state levels of htt. Therefore, we explored the alternate possibility that longer polyQ stretches in htt impart a tonically increased risk of neuronal death by preferentially increasing the abundance of a toxic isoform of the protein over less toxic isoforms. We previously established that monoclonal antibody 3B5H10 recognizes a compact, two-stranded, hairpin conformation of htt that strongly predicts when neurons will die (J. Miller, M. Arrasate, C. Peters-Libeu, and S. Finkbeiner, unpublished observations). Normalized to the total amount of htt ${ }^{\text {exl }}$ $\mathrm{Q}_{n}$-eGFP in neurons, neurons transfected with longer polyQ stretch htt had significantly more $3 \mathrm{~B} 5 \mathrm{H} 10$ conformer (Fig. $2 d$ ). Estimates of the absolute abundance of the $3 \mathrm{~B} 5 \mathrm{H} 10$ conformer in neurons (calculated by multiplying the values in Fig. $2 b$ by those in Fig. $2 d$ ) confirmed a direct relationship between conformer 
a

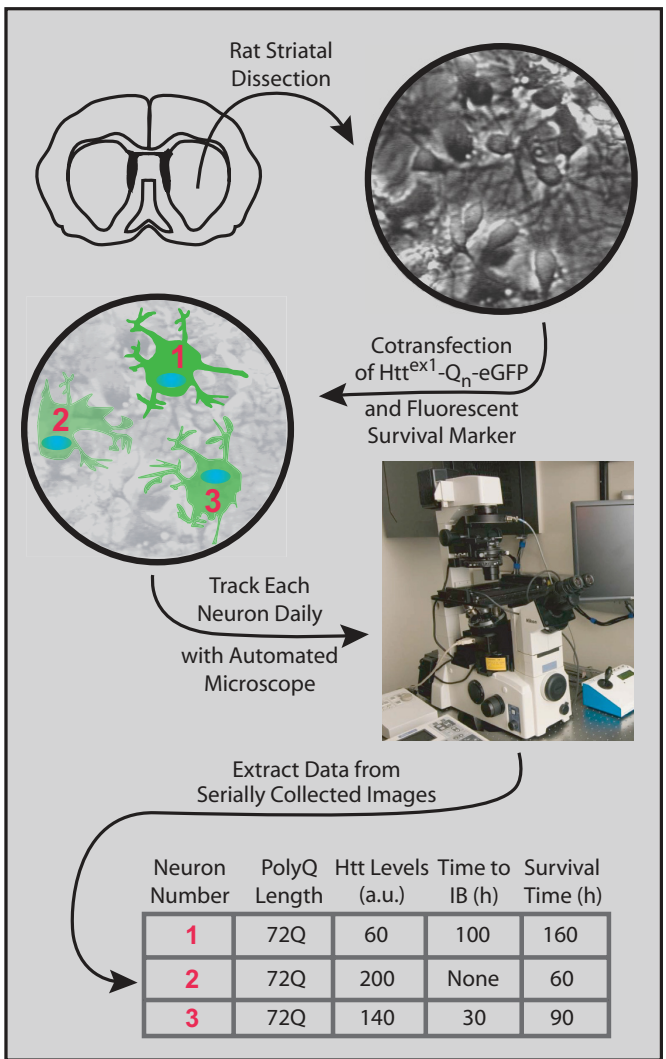

b

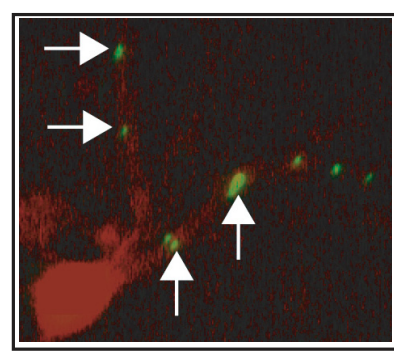

C

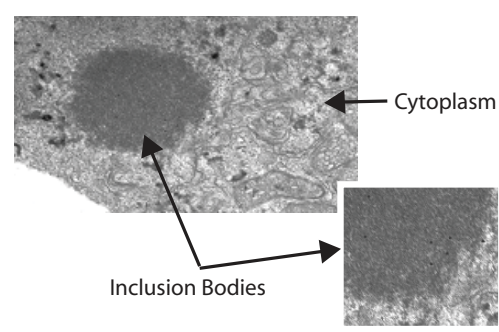

d

STRIATAL NEURON MODEL RECAPITULATES FEATURES OF HD

\begin{tabular}{l}
\multicolumn{1}{c}{ Feature } \\
\hline PolyQ-dependent neuron death
\end{tabular}

\begin{tabular}{ll}
\hline 1 Snell et al., 1993 & 8 Reiner et al., 1988 \\
2 Saudou et al., 1998 & 9 Richfield et al., 1995 \\
3 Dragunow et al., 1995 & 10 Sapp et al., 1995 \\
4 Difiglia et al., 1997 & 11 Gutekunst et al., 1999 \\
5 Arrasate et al., 2004 & 12 Sapp et al., 1997 \\
6 Vonsattel et al., 1985 & 13 Sieradzan et al., 1999 \\
7 Myers et al., 1988 & 14 Kuemmerle et al., 1999
\end{tabular}

al., 2009

Unpublished observations

+ Supporting data in this study

\# Dr. Marian DiFiglia (personal communication)

99 OBSERVED IN ANIMAL MODELS OF POLYQ DISORDERS OR IN HD

\begin{tabular}{|c|c|c|}
\hline Feature & Neuron model & Mouse/rat model or HD \\
\hline Manipulation of nuclear localization affects phenotype & & \\
\hline Interference with ubiquitination leads to a reduction in IB formation & & \\
\hline Interference with ubiquitination leads to an exacerbation of patholo & & \\
\hline Ciliary neurotrophic factor suppresses neurodegeneration & & \\
\hline $\mathrm{Htt}$ contains an Akt site and is phosphorylated at ser- 421 in neurons & & \\
\hline $\mathrm{Htt}$ phosphorylation (S-421) is polyQ-dependent & & \\
\hline IB formation reduces intracellular levels of polyQ-expanded protein & & \\
\hline Accelerated IB formation can be associated with improved outcome & & \\
\hline
\end{tabular}

$\begin{array}{ll}\text { 1 Saudou et al., 1998 } & \text { 7 Warby et al., 2005 } \\ \text { 2 Schilling et al., 2004 } & \text { 8 Arrasate et al., 2004 } \\ \text { 3 Benn et al., 2005 } & \text { 9 Mitra et al., 2009 } \\ \text { 4 Cummings et al., 1999 } & \text { 10 Kuemmerle et al., 1999 } \\ \text { 5 Zala et al., 2004 } & \text { 11 Gutekunst et al., 1999 } \\ \text { 6 Humbert et al., 2002 } & \text { 12 Slow et al., 2005 }\end{array}$

* Unpublished observations

\# Dr. Huda Zoghbi (personal communication) 
a

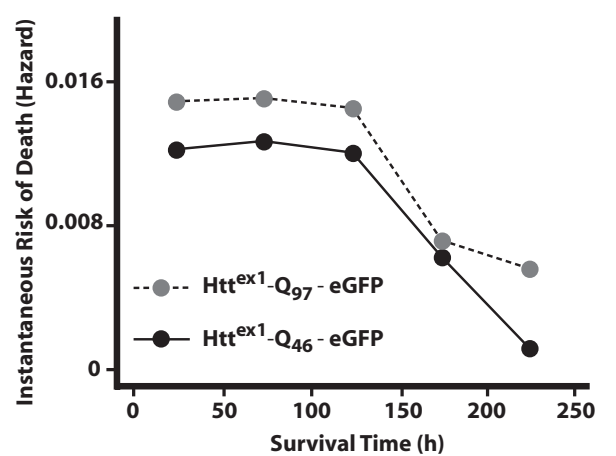

b

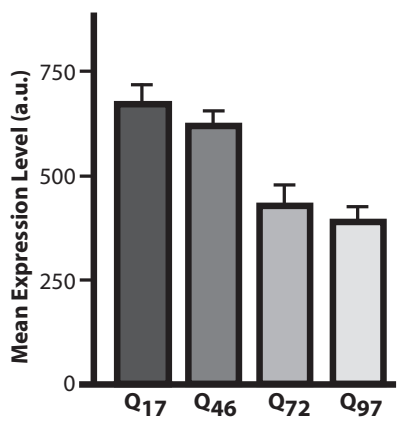

d

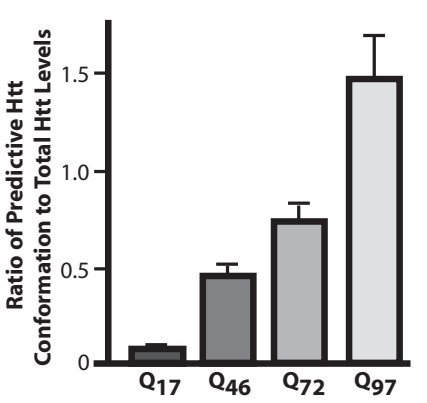

Figure 2. Expanded polyQ results in an initially constant, then decreasing, rate of death, decreased htt expression, and increases in a specific htt conformation. $\boldsymbol{a}$, Risk of death (graphed as instantaneous hazard in actuarial survival analysis) was plotted against survival time for a cohort of striatal neurons transfected with htt ${ }^{\mathrm{ex} 1}-\mathrm{Q}_{46}$-eGFP and another cohort transfected with htt ${ }^{\mathrm{ex} 1}-\mathrm{Q}_{97}$-eGFP. $\boldsymbol{b}$, Increasing polyQ length results in lower htt expression levels. Mean diffuse htt expression level (in arbitrary units of fluorescence) for htt ${ }^{\mathrm{ex}}$ $Q_{17,46,72, \text { or } 97}$-eGFP-transfected neurons, measured with automated microscopy at $24 \mathrm{~h}$ after transfection. Neurons that form IBs by $24 \mathrm{~h}$ are excluded from the analysis. $c$, Normalized diffuse $\mathrm{htt}$ expression levels for neurons fixed $24 \mathrm{~h}$ after transfection. For each neuron, levels of diffuse htt, estimated from htt-eGFP fluorescence, were normalized to the fluorescence of the transfection marker mRFP to control for differences in transfection and transcriptional activation. $\boldsymbol{d}$, Increasing polyQ length preferentially leads to an increase in the abundance of a htt conformer that strongly predicts neuronal death. Neurons transfected with htt ${ }^{\text {ex }}-Q_{17,46}, 72$, or 97 -eGFP were fixed at $24 \mathrm{~h}$ and immunostained with a monoclonal antibody, 3B5H10, which recognizes a conformation of htt that predicts neuronal death. Levels of antibody staining in each neuron were quantified via immunofluorescence and normalized to the htt levels of that neuron, as measured by eGFP fluorescence. An analysis of absolute levels of 3B5H10 conformer (not normalized to htt levels) confirmed the trends in $\boldsymbol{d}$ (data not shown). All error bars are $95 \%$ confidence intervals.

cidated between levels of diffuse mutant htt and neuronal death. We therefore measured htt ${ }^{\text {exl }}-\mathrm{Q}_{46}$-eGFP levels in thousands of individual striatal neurons $24 \mathrm{~h}$ after transfection and followed each neuron until its death. We then analyzed the dataset with one of the most widely used regression techniques in clinical medicine for analyzing the relationship between risk factors and time to death, the Cox proportional hazard model.

In our model, we treated time to death as the outcome and htt levels as the independent (predictor) variable. We stratified our analysis by experiment date to control for any experiment-toexperiment variability in survival. Because we previously showed that higher $\mathrm{htt}^{\mathrm{exl}}-\mathrm{Q}_{46}$-eGFP levels lead to earlier IB formation (which dramatically changes subsequent survival time) (Arrasate et al., 2004), we were concerned whether the formation of an IB could confound our analysis of diffuse htt levels and survival times. We therefore also stratified our analysis by IB status (neurons that never formed an IB during the experiment versus neurons that did). Finally, our Cox model used a technique (called penalized splines) that allows a more flexible regression between htt levels and time to death. This allowed us to test for nonlinear relationships between htt levels and risk of death.

Similar to our previous result (Arrasate et al., 2004), Cox regression revealed that neurons with the highest levels of htt ${ }^{\text {exl }}$ -
$\mathrm{Q}_{46}$-eGFP had the highest risk of death (Fig. 3). Repeating the Cox analysis on only the cohort of neurons that never formed an IB during the experiment yielded highly similar results (data not shown).

To confirm that the effect of htt ${ }^{\text {exl }}$ $\mathrm{Q}_{46}$-eGFP levels on life span was specific, we repeated the experiment on neurons transfected with htt ${ }^{\mathrm{exl}}-\mathrm{Q}_{17^{-}}$ eGFP or eGFP-only (Fig. 3). In the case of htt ${ }^{\text {ex } 1}-Q_{17}$-eGFP-transfected neurons, there was no statistically significant relationship between expression levels and survival. For neurons transfected with eGFP alone, regression demonstrated a nonlinear, shallow, U-shaped curve for the relationship between expression and death (Fig. 3). At expression levels generally seen in htt-transfected neurons, eGFP-only predicted improved survival. At expression levels higher than those seen in htt-transfected neurons, eGFP-only predicted higher risk of death. Thus, the inverse relationship between htt levels and survival is specific to disease-associated versions of htt.

The quantitative relationship between mutant htt levels and neuronal death provides a basis to evaluate other proposed mechanisms of polyQ toxicity. In one model, putative toxic species of mutant htt lead to neurodegeneration by interacting with one or more selected intracellular targets and modulating their function in a deleterious manner (Schaffar et al., 2004; Gidalevitz et al., 2006). In another model, malfolded proteins form oligomeric pore-like structures that cause neurodegeneration by punching holes in membranes (Caughey and Lansbury, 2003; Kayed et al., 2004; Glabe, 2006). These two models make different predictions about the relationship between mutant htt levels and neuronal death. Neurodegeneration caused by interaction between a mutant htt species and a limited number of intracellular protein targets should show a saturable dose-response relationship. However, membrane-based mechanisms of action, such as pore formation, would not be expected to saturate. We found that the relationship between htt levels and neuronal death saturates at long polyQ lengths. For htt ${ }^{\mathrm{ex} 1}-\mathrm{Q}_{72}$-eGFP-transfected neurons, there is a diminished but still significant relationship between htt levels and risk of death (Fig. 3). Neurons transfected with htt ${ }^{\text {exl }}-\mathrm{Q}_{97}-\mathrm{eGFP}$ show no statistically significant relationship between expression and risk of death (Fig. 3). Similar trends were again found when the Cox analysis was applied only to the cohort of neurons that never formed an IB during the experiment (data not shown). We also observed that the relationship between htt levels and IB formation saturates at long polyQ lengths (data not shown). These findings suggest that mutant htt pathogenesis may occur via interactions with a specific and limited set of cellular targets and that the process of IB formation is a regulated cellular process governed by saturable molecular mechanisms. 

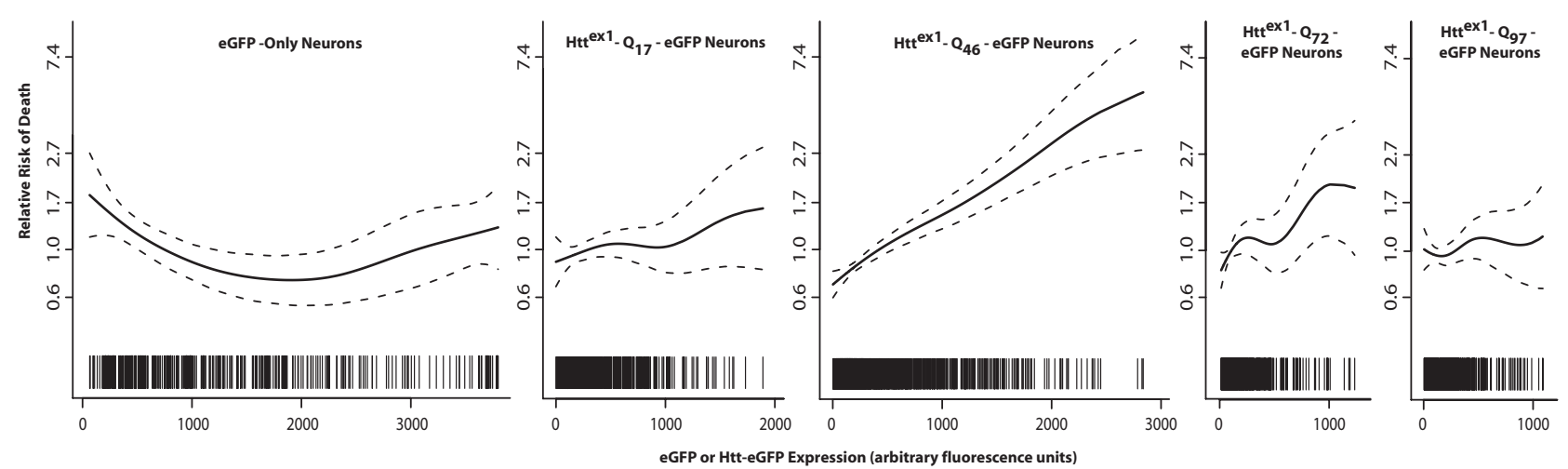

Figure 3. Saturable relationship between htt levels and neuronal death. Relative risk of death versus expression levels for eGFP or htt ${ }^{\text {ex1 }}-Q_{17,46,72, \text { or } 97}$-eGFP-transfected striatal neurons. The dotted lines represent SE bands. The vertical hash marks represent the htt levels for individual neurons in each dataset. Because the Cox analysis for each graph was performed separately, comparisons of absolute risk between graphs is not possible. The $x$-axes were slightly truncated for the htt ${ }^{\text {ex1 }}-0_{17,46,72}$, or 97 -eGFP graphs because error bands became very large $(>1$ log unit of relative risk). Cox relative risk estimators for each dataset are shown in Table 1.

Table 1. Cox relative risk estimators for the effects of htt concentration on neuronal death, analyzed by polyQ length

\begin{tabular}{llll}
\hline Construct & $\begin{array}{l}\text { Linear } \\
\text { component }\end{array}$ & $p$ value & $\begin{array}{l}\text { Nonlinear component } \\
p \text { value }\end{array}$ \\
\hline eGFP-only & -0.00006384 & 0.26 & $\mathbf{0 . 0 0 3 6}$ \\
$Q_{17}$ & 0.0002130 & 0.095 & 0.80 \\
$Q_{46}$ & 0.0006906 & $<10^{-99}$ & 0.21 \\
$Q_{72}$ & 0.0006048 & $\mathbf{0 . 0 0 7 0}$ & 0.29 \\
$Q_{97}$ & 0.0003609 & 0.070 & 0.42 \\
\hline
\end{tabular}

Relative risk estimators were derived from the datasets used to produce Fig. 3.

Table 2. Comparison of disease progression phenotypes in HD homozygous versus long polyQ patients

\begin{tabular}{lll}
\hline Feature & $\begin{array}{l}\text { Homozygous } \\
\text { patients }\end{array}$ & $\begin{array}{l}\text { Long polyQ } \\
\text { patients }\end{array}$ \\
\hline $\begin{array}{c}\text { Parkisonism and other presenting motor } \\
\text { symptoms atypical of "classic" HD }\end{array}$ & $\sqrt{ }^{a}$ & $\sqrt{ }^{b, c, d}$ \\
$\begin{array}{c}\text { Progression of disease from age of onset to death } \\
\text { faster than typical HD patients }\end{array}$ & $\sqrt{ }^{a}$ & $\sqrt{ }^{c, e, f, g}$ \\
$\begin{array}{c}\text { Brain atrophy more widespread than typical HD } \\
\text { (including cerebellar degeneration) }\end{array}$ & $\sqrt{ }^{a}$ & $\sqrt{ }^{b, c, e, h}$ \\
\hline
\end{tabular}

Evidence from $\mathrm{HD}$ clinical data consistent with the mechanism of polyQ-dependent toxicity in disease progression being saturable. Because toxicity is saturable in our simple HD model (Fig. 3), we reasoned that patients with a homozygous gene dosage of mutant htt in the $\mathrm{Q}_{40-50}$ range and patients with a heterozygous gene dosage of mutant htt in the long polyQ range may also demonstrate saturated toxicity, causing these two, distinct patient populations to partially phenocopy each other. Reexamining the clinical HD literature, we found that both homozygous HD patients and long polyQ expansion heterozygous patients (even those without juvenile-onset HD) deviate in similar ways from a classic HD disease progression phenotype. However, these two patient populations do not have a similar age-at-symptom onset, suggesting that the mechanism of polyQ-dependent toxicity in disease progression, but not disease onset, is saturable.

${ }^{a}$ Squitieri et al., 2003.

${ }^{b}$ Seneca et al., 2004.

'Squitieri et al., 2000.

${ }^{d}$ Squitieri et al., 2006.

${ }^{e}$ Ruocco et al., 2008.

'Rosenblatt et al., 2006

${ }^{g}$ Ravina et al., 2008.

${ }^{h}$ Vonsattel and DiFiglia, 1998.

\section{Different conformational changes in monomeric htt are the} rate-limiting steps for IB formation and neuronal death Having quantified an inverse but saturable relationship between mutant $h t t^{\text {exl }}-\mathrm{Q}_{n}$-eGFP levels and neuronal survival, we next asked whether IB formation exhibited a quantitatively similar htt concentration dependence. This relationship has been used to deduce whether the rate-limiting step in IB formation involves structural changes to a monomer of htt or the formation of a dimer. Wetzel and colleagues found that polyQ polymerization into amyloid fibrils in vitro has a first-order dependence on polyQ peptide concentration, and they therefore concluded that the rate-limiting step in amyloid formation is the unimolecular conformation change of polyQ into a nucleus that can seed aggregation (Chen et al., 2002). In contrast to Wetzel, Wittrup and colleagues showed that IB formation in an immortalized striatal line transfected with mutant $h \mathrm{tt}^{\mathrm{ex} 1}$ may have a second-order dependence on htt concentration, suggesting that the rate-limiting step in IB formation is the collision of two htt molecules (Colby et al., 2006).

To compare the htt concentration dependence for neuronal death and IB formation, we followed striatal neurons transfected with $h t t^{\text {ex }}-\mathrm{Q}_{46}-\mathrm{eGFP}$, measuring average diffuse htt levels for each neuron at $24 \mathrm{~h}$ and then noting the time of IB formation and/or time of death of the neuron. Neurons that had already formed an IB at $24 \mathrm{~h}$ were excluded from the analysis.

To analyze these datasets, we sought a regression technique that allowed us to flexibly model the linear and nonlinear terms in the relationship between htt levels and IB formation or death. Since we can only measure IB formation and death at each imaging time point, we only know that a neuron formed an IB or died in a given interval (the interval between imaging time points) rather than at a specific time. Thus, the regression technique we used also needs to accommodate such interval-censored data. Additionally, because there may be some variability in overall rates of IB formation or death from experiment to experiment, we sought a regression technique that could control for such variability. Finally, to comport our data with classic rate equation analysis, we needed a regression technique that transforms the outcome we can measure (time to IB formation or time to death) into a scale that is directly proportional with rate of IB formation or death. A Weibull accelerated failure time regression model with interval censoring and penalized spline treatment of htt levels meets all of these criteria.

Weibull regression analysis of the datasets revealed a primarily first-order relationship between htt levels and the rates of both death and IB formation (Fig. 4a,b), consistent with a model in which a change in the conformation of single molecules of mutant htt may be the rate-limiting steps for both neuronal death and IB formation.

Interestingly, as htt concentration increases, the rate of IB formation increases faster than the rate of death (Fig. 4c). This 


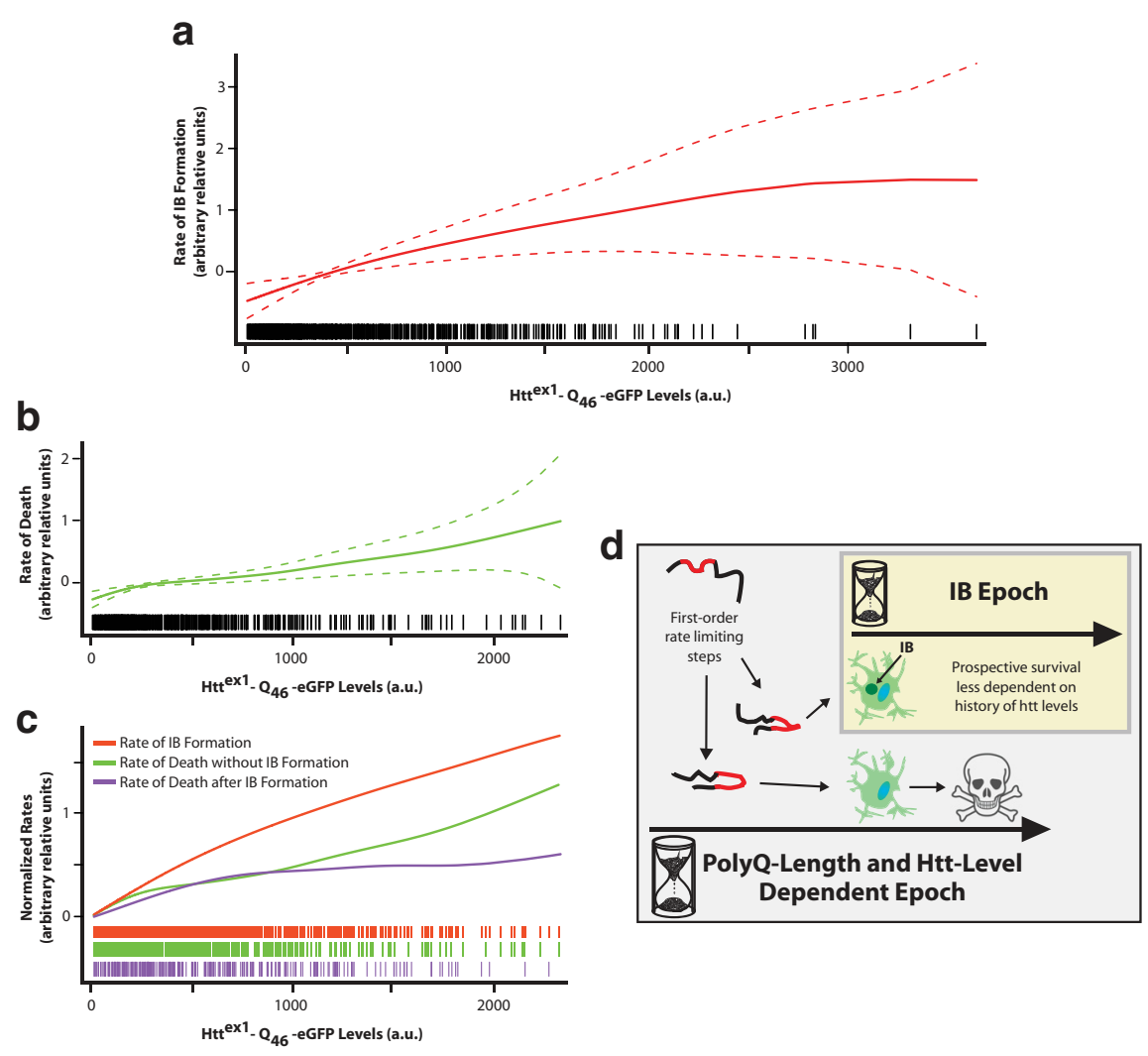

Figure 4. Different conformational changes in monomeric htt may be the rate-limiting steps for neuronal death and a protective response marked by IB formation. $\boldsymbol{a}$, Rate of IB formation has a primarily first-order dependence on $\mathrm{htt}^{\mathrm{e}{ }^{\mathrm{e} 1}-\mathrm{Q}_{46}}{ }^{-\mathrm{eGFP}}$ concentration. Linear component of the fitted regression, $p<10^{-99}$; nonlinear component to fitted regression, $p=0.005$. The nonlinear component to the regression is clearly below first-order, as demonstrated by the decelerating slope of the regression line. The dotted lines represent SE bands. The vertical hash marks represent the htt levels for individual neurons in the dataset. $\boldsymbol{b}$, Rate of death has a first-order dependence on $\mathrm{htt}^{\mathrm{ex} 1}-\mathrm{Q}_{46}$-eGFP concentration. Linear component of the fitted regression, $p=2.9 \times 10^{-12}$; nonlinear component to fitted regression, $p=0.22$. SE bands and vertical hash marks are as in $\boldsymbol{a}$. c, Comparison of htt concentration dependence for the rates of IB formation (red), death without IB formation (green), and death after IB formation (purple). To facilitate comparison, $x$-intercepts for each curve have been normalized to 0 . The rate of $I B$ formation has the strongest concentration dependence, followed by the rate of death without IB formation. In contrast, death rates after IB formation have a diminished dependence on pre-IB htt levels, suggesting the neuron has entered an adapted epoch. The colored vertical hashes represent the htt levels for individual neurons in each respective dataset. $\boldsymbol{d}$, Model of htt molecular pathogenesis consistent with data from Figure 4. Different first-order rate-limiting changes in monomeric htt conformation (polyQ stretch in red) lead to divergent fates for the neuron. One fate involves accelerated neuronal death, in which time to death can be reliably predicted by the combination of the htt levels of the neuron and polyQ length. The other fate involves an adapted epoch, marked by IB formation. Survival time in this epoch has a diminished dependence on the pre-IB htt levels of the neuron.

suggests that the rate-limiting steps for IB formation and death may involve different conformers of monomeric htt.

IB formation heralds an adapted epoch

If a neuron forms an IB, its diffuse mutant htt levels drop to nearly undetectable levels and its survival dramatically improves. However, if the neuron does not form an IB, its risk of death remains tonically high (Arrasate et al., 2004). Given the beneficial effects of IB formation on mutant htt levels and neuronal survival, we have proposed that that IB formation is a coping response that mitigates the effects of toxic conformer(s) of diffuse mutant htt (Arrasate et al., 2004). To test this hypothesis, we measured the time interval from IB formation to death for neurons transfected with mutant htt and investigated whether there was a relationship to the initial htt levels of each neuron. Weibull accelerated failure time regression revealed a decreased htt concentration dependence for the rate of death after a neuron forms an IB (Fig. 4c). Thus, IB formation marks a new adapted era for the neuron in which the history of diffuse htt levels of the neuron has a diminished impact on its instantaneous risk of death.

\section{Discussion}

We designed an automated microscopy system and applied it to a primary striatal neuron model of HD to quantitatively relate htt levels, polyQ length, IB formation, and neuronal death to each other. The validity of this approach toward developing an HD cellular systems model is supported by two properties of our experimental setup. First, the automated microscopy approach involves the tracking of thousands of individual neurons over their entire lifetime and measuring with high sensitivity how well particular observations predict an outcome of interest. Therefore, pitfalls of population-based methods for relating risk factors to outcome can be avoided, namely the loss of data contained in neuron-to-neuron variability. The method also avoids the issue of mistaking cellular adaptive responses for risk factors (and vice versa) associated with snapshot experimental approaches. Second, the striatal model recapitulates key features of $\mathrm{HD}$ and has predicted findings later observed in animal models of polyQ disorders and HD (Fig. 1b-e).

The systems model that emerged from these experiments place critical limits on the potential mechanisms by which mutant htt might induce neuronal death. The constant rate of death in the model up to $6 \mathrm{~d}$ after mutant htt transfection (Fig. 2a) strongly supports a one-hit model of neurodegeneration, in which mutant htt tonically decreases the homeostatic capacity of a neuron. Thus, a single random stress (one-hit), which would normally be withstood by a wild-type neuron, instead triggers death in a neuron with mutant htt. Our data also show that, as the length of the polyQ stretch increases, the risk of neuronal death also increases but that increased risk remains constant over time. This suggests that, as neurons are exposed to htt with progressively longer polyQ stretches, cellular homeostatic capacity decreases further, making them even more susceptible to small, one-hit insults.

Recent analysis of rates of death for a range of neurodegenerative diseases and disease models suggests that the one-hit model of neurodegeneration can be improved with stretched exponential decay models. Such models, which most easily fit data in which the rate of death decreases over time, are consistent with multiple populations of neurons coexisting, each with a different tonic (one-hit) rate of death (Clarke and Lumsden, 2005a,b). Interestingly, beginning $6 \mathrm{~d}$ after transfection, we observed a decrease in the rate of neuronal death for both $\mathrm{htt}^{\mathrm{ex1}}-\mathrm{Q}_{46}-\mathrm{eGFP}$ - and $h t^{e x 1}-Q_{97}-e G F P-t r a n s f e c t e d$ neurons. In both the neuronal 
system used here and in HD patients, striatal neurons that are enkephalin-negative are more resistant to mutant htt-induced toxicity (Fig. 1d). The lower rate of death we observe may be attributable to the rising proportion of enkephalin-negative neurons by $6 \mathrm{~d}$ after transfection. Thus, the drop in rate of death could be consistent with a heterogeneous population of striatal neurons, each with a different constant rate of death (e.g., "heterogeneous population one-hit" model).

Unexpectedly, our striatal model revealed that diffuse htt levels are inversely proportional to polyQ length (Fig. $2 b, c)$. Since all $h t^{\text {exl }}-\mathrm{Q}_{n}$-eGFP constructs used in our striatal model were expressed from the same vector backbone with the same promoter and with irregularly alternating CAG and CAA codons encoding the polyQ stretch, differences in steady-state levels likely arise from differences in htt half-life rather than differences in transcription or message stability. If so, this would suggest that striatal neurons can recognize and efficiently degrade even the longest of polyQ-expanded htt (Michalik and Van Broeckhoven, 2004).

Although increasing polyQ length results in decreased htt expression, longer polyQ stretches increase the absolute abundance of a specific htt conformation (Fig. $2 d$ ). This conformation, recognized by the monoclonal antibody $3 \mathrm{~B} 5 \mathrm{H} 10$, strongly predicts death, contains a compact, two-stranded, hairpin polyQ structure, and is found in monomeric htt (J. Miller, M. Arrasate, C. Peters-Libeu, and S. Finkbeiner, unpublished observations). The hairpin structure of the polyQ stretch in monomeric htt could impart toxicity by providing an accessible protein surface in a conformation that promiscuously binds with a variety of protein partners and alters their function (Schaffar et al., 2004).

For mutant htt with a polyQ stretch of a given length, the HD cellular systems model investigated here demonstrated a significant positive correlation between higher htt levels and both increased toxicity and IB formation. In contrast, neurons transfected with eGFP alone demonstrated a U-shaped correlation between fluorescent protein expression levels and toxicity. The relative risk of death is modestly lower among neurons expressing eGFP at intermediate levels than with neurons expressing eGFP at the lowest or highest levels (Fig. 3). Since our approach is quite sensitive, the biological significance of this fluctuation in survival with eGFP expression levels is not clear. However, over the range of expression levels achieved by $h t^{\mathrm{exl}}-\mathrm{Q}_{n}$-eGFP, in which higher expression leads to greater toxicity, there is an opposite association between survival and eGFP expression levels. This argues even more strongly for the specificity of the expressiondependent mutant htt toxicity we report in Figure 3.

Interestingly, as the polyQ length increased, the incremental effect of mutant htt expression on toxicity and IB formation became smaller, suggesting that toxicity and IB formation are saturable in this model (Fig. 3) (data now shown). Mathematical modeling of data from patients with polyQ-dependent neurodegenerative diseases is consistent with the idea that increases in polyQ protein levels incrementally contribute less to toxicity as polyQ length increases, implying saturable toxicity in human patients as well (Sugaya and Matsubara, 2009). We thus explored whether any observations in the clinical literature would further support the idea of saturable toxicity. We reasoned that two patient populations were most likely to demonstrate saturated toxicity: HD patients who are homozygous for the mutant htt allele and heterozygous HD patients with very long polyQ stretches in their mutant htt allele. We also reasoned if these distinct patient populations did possess saturated toxicity, they may display a common saturated toxicity phenotype. A search of the HD clinical literature revealed phenotypic parallels between these two groups (Table 2). Compared with a classic HD phenotype, both groups of patients demonstrated atypical presenting motor symptoms, including parkinsonism rather than a chorea, faster symptom progression, and more widespread brain atrophy (including cerebellum). These parallels held true even when considering long polyQ heterozygous patients with adult-onset (rather than juvenile-onset) HD.

Although there were phenotypic parallels between these patient populations, there were also key differences. For example, age-at-symptom onset (AAO) for homozygous HD patients is fairly similar to AAO for heterozygous patients with the same polyQ length. In contrast, long polyQ patients clearly have an earlier AAO. Thus, the mechanisms of polyQ toxicity in human patients that lead to symptom onset may not be saturable, whereas the mechanisms of polyQ toxicity that lead to disease progression (phenotypically manifesting as the rate of disease progression, areas of brain atrophy, and types of motor symptoms) may be saturable.

Two competing theories have been advanced for the ratelimiting step in IB formation, one involving a unimolecular change in htt conformation and the other involving a collision between two htt molecules (Chen et al., 2002; Colby et al., 2006). Resolving this debate has implications for therapy. For example, if the rate-limiting step in the mechanism of toxicity of mutant htt involves an analogous collision between two htt molecules, then reducing htt by only a small amount should dramatically decrease toxicity. Alternatively, if the rate-limiting step of toxicity involves an analogous change in monomeric htt conformation, then targeting the equilibrium between correctly folded and misfolded htt monomers (rather than targeting the htt oligomerization process) would potentially be a better therapeutic strategy. A plot of htt levels versus rate of IB formation for striatal neurons transfected with htt ${ }^{\text {ex1 }}-\mathrm{Q}_{46}$-eGFP reveals a predominantly firstorder relationship (Fig. 4a), supporting a unimolecular conformational change in mutant htt as the rate-limiting step toward IB formation.

Although we are uncertain why our data on the htt concentration dependence of IB formation diverges from the studies of Colby et al., we note several differences between the studies. First, their study used an immortalized striatal cell line rather than primary striatal neurons. Furthermore, the data collection portion of their experiments took place on cultures less than a day old, compared with the relatively mature primary culture used in our studies. Additionally, whereas they sorted cells into 8-15 concentration categories and measured the number of IBs in each category, our experiments allowed us to consider every single cell separately over its entire lifetime, providing thousands of temporal datapoints with which to carry out a more data-rich regression analysis on htt levels versus rate of IB formation.

Although the htt concentration dependence for IB formation and neuronal death were both primarily first-order, the dependence was greater for IB formation (Fig. 4c). Thus, as htt concentration increases, the rate of IB formation accelerates faster than the rate of death. These differences in htt concentration dependence suggest that IB formation and death are triggered by different conformations of monomeric mutant htt. Previous studies support the idea of multiple different folding pathways for mutant htt, seeded by different monomeric conformations (Wacker et al., 2004; Legleiter et al., 2009). One pathway may preferentially lead to an adaptive homeostatic response marked by IB formation, whereas another pathway may lead to an elevated risk of neuronal death (Fig. $4 d$ ). These data provide a quantitative rationale for the observation that small-molecule screens targeted at 
decreasing IB formation have often resulted in therapeutic hits, even though IBs may be part of a coping response (Zhang et al., 2005; Desai et al., 2006). For example, if a potential therapeutic leads to a decrease in htt levels, IB formation rates will drop faster than death rates. Alternatively, if a potential therapeutic blocks the improper folding of monomeric htt into multiple conformations, both IB formation and death rates will again drop.

For neurons that trigger IB formation (Fig. $4 d$ ), our data demonstrate that the remaining lifetime of the neuron has a diminished dependence on pre-IB htt levels (Fig. 4c). This suggests that the formation of an IB marks a new adapted epoch for neurons (Fig. 4d). Together with our previous observation that IB formation leads to a precipitous drop in toxic diffuse mutant htt levels (Arrasate et al., 2004), the data here explain why behavioral deficits and pathological changes can be nearly completely reversed after symptoms appear by reducing htt levels (Yamamoto et al., 2000; Harper et al., 2005).

\section{References}

Arrasate M, Finkbeiner S (2005) Automated microscope system for determining factors that predict neuronal fate. Proc Natl Acad Sci U S A 102:3840-3845

Arrasate M, Mitra S, Schweitzer ES, Segal MR, Finkbeiner S (2004) Inclusion body formation reduces levels of mutant huntingtin and the risk of neuronal death. Nature 431:805-810.

Benn CL, Landles C, Li H, Strand AD, Woodman B, Sathasivam K, Li SH, Ghazi-Noori S, Hockly E, Faruque SM, Cha JH, Sharpe PT, Olson JM, Li XJ, Bates GP (2005) Contribution of nuclear and extranuclear polyQ to neurological phenotypes in mouse models of Huntington's disease. Hum Mol Genet 14:3065-3078.

Bennett EJ, Bence NF, Jayakumar R, Kopito RR (2005) Global impairment of the ubiquitin-proteasome system by nuclear or cytoplasmic protein aggregates precedes inclusion body formation. Mol Cell 17:351-365.

Brooks E, Arrasate M, Cheung K, Finkbeiner SM (2004) Using antibodies to analyze polyglutamine stretches. Methods Mol Biol 277:103-128.

Caughey B, Lansbury PT (2003) Protofibrils, pores, fibrils, and neurodegeneration: separating the responsible protein aggregates from the innocent bystanders. Annu Rev Neurosci 26:267-298.

Chen S, Berthelier V, Hamilton JB, O’Nuallain B, Wetzel R (2002) Amyloidlike features of polyglutamine aggregates and their assembly kinetics. Biochemistry 41:7391-7399.

Clarke G, Lumsden CJ (2005a) Heterogeneous cellular environments modulate one-hit neuronal death kinetics. Brain Res Bull 65:59-67.

Clarke G, Lumsden CJ (2005b) Scale-free neurodegeneration: cellular heterogeneity and the stretched exponential kinetics of cell death. J Theor Biol 233:515-525.

Clarke G, Collins RA, Leavitt BR, Andrews DF, Hayden MR, Lumsden CJ, McInnes RR (2000) A one-hit model of cell death in inherited neuronal degenerations. Nature 406:195-199.

Colby DW, Cassady JP, Lin GC, Ingram VM, Wittrup KD (2006) Stochastic kinetics of intracellular huntingtin aggregate formation. Nat Chem Biol 2:319-323.

Cummings CJ, Reinstein E, Sun Y, Antalffy B, Jiang Y, Ciechanover A, Orr HT, Beaudet AL, Zoghbi HY (1999) Mutation of the E6-AP ubiquitin ligase reduces nuclear inclusion frequency while accelerating polyglutamineinduced pathology in SCA1 mice. Neuron 24:879-892.

Desai UA, Pallos J, Ma AA, Stockwell BR, Thompson LM, Marsh JL, Diamond MI (2006) Biologically active molecules that reduce polyglutamine aggregation and toxicity. Hum Mol Genet 15:2114-2124.

DiFiglia M, Sapp E, Chase KO, Davies SW, Bates GP, Vonsattel JP, Aronin N (1997) Aggregation of huntingtin in neuronal intranuclear inclusions and dystrophic neurites in brain. Science 277:1990-1993.

Dominici F, Peng RD, Ebisu K, Zeger SL, Samet JM, Bell ML (2007) Does the effect of PM10 on mortality depend on PM nickel and vanadium content? A reanalysis of the NMMAPS data. Environ Health Perspect 115:1701-1703.

Dragunow M, Faull RL, Lawlor P, Beilharz EJ, Singleton K, Walker EB, Mee E (1995) In situ evidence for DNA fragmentation in Huntington's disease striatum and Alzheimer's disease temporal lobes. Neuroreport 6:1053-1057.
Finkbeiner S, Cuervo AM, Morimoto RI, Muchowski PJ (2006) Diseasemodifying pathways in neurodegeneration. J Neurosci 26:10349-10357.

Gidalevitz T, Ben-Zvi A, Ho KH, Brignull HR, Morimoto RI (2006) Progressive disruption of cellular protein folding in models of polyglutamine diseases. Science 311:1471-1474.

Glabe CG (2006) Common mechanisms of amyloid oligomer pathogenesis in degenerative disease. Neurobiol Aging 27:570-575.

Gutekunst CA, Li SH, Yi H, Mulroy JS, Kuemmerle S, Jones R, Rye D, Ferrante RJ, Hersch SM, Li XJ (1999) Nuclear and neuropil aggregates in Huntington's disease: relationship to neuropathology. J Neurosci 19:2522-2534.

Harper SQ, Staber PD, He X, Eliason SL, Martins IH, Mao Q, Yang L, Kotin RM, Paulson HL, Davidson BL (2005) RNA interference improves motor and neuropathological abnormalities in a Huntington's disease mouse model. Proc Natl Acad Sci U S A 102:5820-5825.

Humbert S, Bryson EA, Cordelières FP, Connors NC, Datta SR, Finkbeiner S, Greenberg ME, Saudou F (2002) The IGF-1/Akt pathway is neuroprotective in Huntington's disease and involves huntingtin phosphorylation by Akt. Dev Cell 2:831-837.

Kayed R, Sokolov Y, Edmonds B, McIntire TM, Milton SC, Hall JE, Glabe CG (2004) Permeabilization of lipid bilayers is a common conformationdependent activity of soluble amyloid oligomers in protein misfolding diseases. J Biol Chem 279:46363-46366.

Kuemmerle S, Gutekunst CA, Klein AM, Li XJ, Li SH, Beal MF, Hersch SM, Ferrante RJ (1999) Huntingtin aggregates may not predict neuronal death in Huntington's disease. Ann Neurol 46:842-849.

Legleiter J, Lotz GP, Miller J, Ko J, Ng C, Williams GL, Finkbeiner S, Patterson PH, Muchowski PJ (2009) Monoclonal antibodies recognize distinct conformational epitopes formed by polyglutamine in a mutant huntingtin fragment. J Biol Chem 284:21647-21658.

Michalik A, Van Broeckhoven C (2004) Proteasome degrades soluble expanded polyglutamine completely and efficiently. Neurobiol Dis 16:202-211.

Mitra S, Tsvetkov AS, Finkbeiner S (2009) Single neuron ubiquitinproteasome dynamics accompanying inclusion body formation in Huntington's disease. J Biol Chem 284:4398-4403.

Myers RH, Vonsattel JP, Stevens TJ, Cupples LA, Richardson EP, Martin JB, Bird ED (1988) Clinical and neuropathologic assessment of severity in Huntington's disease. Neurology 38:341-347.

Nagai Y, Inui T, Popiel HA, Fujikake N, Hasegawa K, Urade Y, Goto Y, Naiki $\mathrm{H}$, Toda $\mathrm{T}$ (2007) A toxic monomeric conformer of the polyglutamine protein. Nat Struct Mol Biol 14:332-340.

Orr HT, Zoghbi HY (2007) Trinucleotide repeat disorders. Annu Rev Neurosci 30:575-621.

Ravina B, Romer M, Constantinescu R, Biglan K, Brocht A, Kieburtz K, Shoulson I, McDermott MP (2008) The relationship between CAG repeat length and clinical progression in Huntington's disease. Mov Disord 23:1223-1237.

Reiner A, Albin RL, Anderson KD, D'Amato CJ, Penney JB, Young AB (1988) Differential loss of striatal projection neurons in Huntington disease. Proc Natl Acad Sci U S A 85:5733-5737.

Richfield EK, Maguire-Zeiss KA, Vonkeman HE, Voorn P (1995) Preferential loss of preproenkephalin versus preprotachykinin neurons from the striatum of Huntington's disease patients. Ann Neurol 38:852-861.

Rosenblatt A, Liang KY, Zhou H, Abbott MH, Gourley LM, Margolis RL, Brandt J, Ross CA (2006) The association of CAG repeat length with clinical progression in Huntington disease. Neurology 66:1016-1020.

Ruocco HH, Bonilha L, Li LM, Lopes-Cendes I, Cendes F (2008) Longitudinal analysis of regional gray matter loss in Huntington disease: effects of the length of the expanded CAG repeat. J Neurol Neurosurg Psychiatry 79:130-135.

Sapp E, Ge P, Aizawa H, Bird E, Penney J, Young AB, Vonsattel JP, DiFiglia M (1995) Evidence for a preferential loss of enkephalin immunoreactivity in the external globus pallidus in low grade Huntington's disease using high resolution image analysis. Neuroscience 64:397-404.

Sapp E, Schwarz C, Chase K, Bhide PG, Young AB, Penney J, Vonsattel JP, Aronin N, DiFiglia M (1997) Huntingtin localization in brains of normal and Huntington's disease patients. Ann Neurol 42:604-612.

Saudou F, Finkbeiner S, Devys D, Greenberg ME (1998) Huntingtin acts in the nucleus to induce apoptosis, but death does not correlate with the formation of intranuclear inclusions. Cell 95:55-66.

Schaffar G, Breuer P, Boteva R, Behrends C, Tzvetkov N, Strippel N, Sakahira 
H, Siegers K, Hayer-Hartl M, Hartl FU (2004) Cellular toxicity of polyglutamine expansion proteins: mechanism of transcription factor deactivation. Mol Cell 15:95-105.

Schilling G, Savonenko AV, Klevytska A, Morton JL, Tucker SM, Poirier M, Gale A, Chan N, Gonzales V, Slunt HH, Coonfield ML, Jenkins NA, Copeland NG, Ross CA, Borchelt DR (2004) Nuclear-targeting of mutant huntingtin fragments produces Huntington's disease-like phenotypes in transgenic mice. Hum Mol Genet 13:1599-1610.

Seneca S, Fagnart D, Keymolen K, Lissens W, Hasaerts D, Debulpaep S, Desprechins B, Liebaers I, De Meirleir L (2004) Early onset Huntington disease: a neuronal degeneration syndrome. Eur J Pediatr 163:717-721.

Sieradzan KA, Mechan AO, Jones L, Wanker EE, Nukina N, Mann DM (1999) Huntington's disease intranuclear inclusions contain truncated, ubiquitinated huntingtin protein. Exp Neurol 156:92-99.

Slow EJ, Graham RK, Osmand AP, Devon RS, Lu G, Deng Y, Pearson J, Vaid K, Bissada N, Wetzel R, Leavitt BR, Hayden MR (2005) Absence of behavioral abnormalities and neurodegeneration in vivo despite widespread neuronal huntingtin inclusions. Proc Natl Acad Sci U S A 102:1140211407.

Snell RG, MacMillan JC, Cheadle JP, Fenton I, Lazarou LP, Davies P, MacDonald ME, Gusella JF, Harper PS, Shaw DJ (1993) Relationship between trinucleotide repeat expansion and phenotypic variation in Huntington's disease. Nat Genet 4:393-397.

Squitieri F, Berardelli A, Nargi E, Castellotti B, Mariotti C, Cannella M, Lavitrano ML, de Grazia U, Gellera C, Ruggieri S (2000) Atypical movement disorders in the early stages of Huntington's disease: clinical and genetic analysis. Clin Genet 58:50-56.

Squitieri F, Gellera C, Cannella M, Mariotti C, Cislaghi G, Rubinsztein DC, Almqvist EW, Turner D, Bachoud-Lévi AC, Simpson SA, Delatycki M, Maglione V, Hayden MR, Donato SD (2003) Homozygosity for CAG mutation in Huntington disease is associated with a more severe clinical course. Brain 126:946-955.

Squitieri F, Frati L, Ciarmiello A, Lastoria S, Quarrell O (2006) Juvenile
Huntington's disease: does a dosage-effect pathogenic mechanism differ from the classical adult disease? Mech Ageing Dev 127:208-212.

Sugaya K, Matsubara S (2009) Nucleation of protein aggregation kinetics as a basis for genotype-phenotype correlations in polyglutamine diseases. Mol Neurodegener 4:29.

Vonsattel JP, DiFiglia M (1998) Huntington disease. J Neuropathol Exp Neurol 57:369-384.

Vonsattel JP, Myers RH, Stevens TJ, Ferrante RJ, Bird ED, Richardson EP Jr (1985) Neuropathological classification of Huntington's disease. J Neuropathol Exp Neurol 44:559-577.

Wacker JL, Zareie MH, Fong H, Sarikaya M, Muchowski PJ (2004) Hsp70 and $\mathrm{Hsp} 40$ attenuate formation of spherical and annular polyglutamine oligomers by partitioning monomer. Nat Struct Mol Biol 11:1215-1222.

Warby SC, Chan EY, Metzler M, Gan L, Singaraja RR, Crocker SF, Robertson HA, Hayden MR (2005) Huntingtin phosphorylation on serine 421 is significantly reduced in the striatum and by polyglutamine expansion in vivo. Hum Mol Genet 14:1569-1577.

Yamamoto A, Lucas JJ, Hen R (2000) Reversal of neuropathology and motor dysfunction in a conditional model of Huntington's disease. Cell 101:57-66.

Yoo SY, Pennesi ME, Weeber EJ, Xu B, Atkinson R, Chen S, Armstrong DL, Wu SM, Sweatt JD, Zoghbi HY (2003) SCA7 knockin mice model human SCA7 and reveal gradual accumulation of mutant ataxin-7 in neurons and abnormalities in short-term plasticity. Neuron 37:383-401.

Zala D, Bensadoun JC, Pereira de Almeida L, Leavitt BR, Gutekunst CA, Aebischer P, Hayden MR, Déglon N (2004) Long-term lentiviralmediated expression of ciliary neurotrophic factor in the striatum of Huntington's disease transgenic mice. Exp Neurol 185:26-35.

Zhang X, Smith DL, Meriin AB, Engemann S, Russel DE, Roark M, Washington SL, Maxwell MM, Marsh JL, Thompson LM, Wanker EE, Young AB, Housman DE, Bates GP, Sherman MY, Kazantsev AG (2005) A potent small molecule inhibits polyglutamine aggregation in Huntington's disease neurons and suppresses neurodegeneration in vivo. Proc Natl Acad Sci U S A 102:892-897. 